\title{
Existence of Order-Preserving Functions for Nontotal Fuzzy Preference Relations under Decisiveness
}

\author{
Paolo Bevilacqua ${ }^{1}$ (1) , Gianni Bosi ${ }^{2, *, t}$ (i) and Magalì Zuanon ${ }^{3}$ \\ 1 Dipartimento di Ingegneria e Architettura, Università di Trieste, 34127 Trieste, Italy; \\ paolo.bevilacqua@dia.units.it \\ 2 Dipartimento di Scienze Economiche, Aziendali, Matematiche e Statistiche "Bruno de Finetti", \\ Università di Trieste, 34127 Trieste, Italy \\ 3 Dipartimento di Economia e Management, Università di Brescia, 25122 Brescia, Italy; \\ magali.zuanon@unibs.it \\ * Correspondence: gianni.bosi@deams.units.it; Tel.: +39-040-558-7115 \\ † Current address: via Università 1,34123 Trieste, Italy.
}

Received: 6 October 2017; Accepted: 26 October 2017; Published: 28 October 2017

\begin{abstract}
Looking at decisiveness as crucial, we discuss the existence of an order-preserving function for the nontotal crisp preference relation naturally associated to a nontotal fuzzy preference relation. We further present conditions for the existence of an upper semicontinuous order-preserving function for a fuzzy binary relation on a crisp topological space.
\end{abstract}

Keywords: fuzzy binary relation; fuzzy Suzumura consistency; order-preserving function; maximal element

\section{Introduction}

The literature concerning the (continuous) real representation of a fuzzy binary relation $R: X \times X \mapsto[0,1]$ is concentrated on the cases of fuzzy total preorders (see e.g., Billot [1], Fono and Andjiga [2], Fono and Salles [3], and Agud et al. [4]), and respectively fuzzy interval orders (see e.g., Mishra et Srivastava [5]). For any two points $x, y \in X, R(x, y)$ is interpreted as "the degree to which the alternative $x \in X$ is at least as good as the alternative $y \in X$ ", or equivalently as "the degree to which $x \in X$ is weakly preferred to $y \in X$ ".

We recall that a fuzzy binary relation is said to be total (or connected, or else complete) if $R(x, y)+$ $R(y, x) \geq 1$ for all $x, y \in X$. Some authors call "complete" a fuzzy binary relation satisfying the stronger property according to which $\max (R(x, y), R(y, x))=1$ for all $x, y \in X$ (see e.g., Beg et Ashraf [6]). Such definitions of completeness clearly correspond to the concept of completeness of a crisp binary relation $\succsim$ (a particular case of a fuzzy binary relation $R$ such that $R(x, y) \in\{0,1\}$ for all $x, y \in X$ ). Clearly, the existence of a utility function for a fuzzy binary relation $R: X \times X \mapsto[0,1]$ (i.e., $u$ is a real-valued function on $X$ such that $R(x, y) \geq R(y, x)$ is equivalent to $u(x) \geq u(y)$ for all $x, y \in X)$ is strictly related to different definitions of transitivity of $R$ (like bin-transitivity, min-transitivity, and product transitivity, among the others).

On the other hand, it should be noted that there is a large number of contributions to the representation of nontotal binary relations $\succsim$ (in particular nontotal preorders) in the crisp case (see e.g., Chapter 5 in Bridges amd Mehta [7], Herden and Levin [8], Herden [9], Mehta [10], Bosi et al. [11], Bosi and Mehta [12], and Bosi and Zuanon [13]). This is the case when there are elements $x, y \in X$ such that neither $x \succsim y$ nor $y \succsim x$. In this case a utility function (i.e., a real-valued function $u$ on $X$ such that $x \succsim y$ is equivalent to $u(x) \geq u(y)$ for all $x, y \in X)$ cannot exist, and one can only ask for the existence of an order-preserving function (i.e., of a real-valued function $u$ on $X$ such that, for all $x, y \in X, x \succsim y$ implies $u(x) \geq u(y)$, and $x \succ y$ implies $u(x)>u(y)$, where $\succ$ stands for the strict part of $\succsim$ ). 
While the literature is mainly concerned with the existence of order-preserving functions for not necessarily total preorders (i.e., for reflexive and transitive binary relation), it should be noted that the existence of an order-preserving function for a binary relation $\succsim$ on a set $X$ does not imply the transitivity of $\succsim$, but only the property called Suzumura consistency (see Suzumura [14]), according to which, for example, $(x \succsim y)$ and $(y \succsim z)$ imply not $(z \succ x)$ for all $x, y, z \in X$. This property is much weaker than transitivity, which-as it is well known-requires that, for all $x, y, z \in X, x \succsim z$ whenever $(x \succsim y)$ and $(y \succsim z)$. Suzumura introduced this sort of consistency in order to generalize the Szpilrajn theorem (see Szpilrajn [15]), and, for example, Bosi and Herden [16]), according to which every (pre)order admits an extension by means of a total (pre)order (see also Cato [17]).

In this paper, we present some initial results concerning the existence of an order-preserving function $u:(X, R) \mapsto(\mathbb{R}, \geq)$ for a nontotal fuzzy binary relation $R$ on a set $X$ under decisiveness. This means that we look for the existence of a real-valued function $u$ on $X$ such that for all $(x, y) \in X \times X$ with $R(x, y)+R(y, x) \geq 1, R(x, y) \geq R(y, x)$ implies $u(x) \geq u(y)$, and respectively $R(x, y)>R(y, x)$ implies $u(x)>u(y)$. We refer to decisiveness since the presence of a fuzzy binary relation which is nontotal in our sense is related to the existence of pairs $(x, y) \in X \times X$ such that $R(x, y)+R(y, x)<1$. In other cases, the representation of nontotal (or else partial) (fuzzy) binary relations is performed by allowing partial definition of some utility representation functions (see e.g., Bosi et al. [18]) or of the fuzzy binary relation $R$ itself (see e.g., Khalid and Beg [19]).

It is clear that if $R$ is total in the sense above, then an order-preserving function $u$ is a utility for $R$, and this clearly implies that every result concerning the existence of an order-preserving function for a fuzzy binary relation generalizes the corresponding result for a fuzzy total binary relation, where "corresponding" essentially means "under the same assumptions of transitivity of $R$ ".

We use this kind of argument in order to present conditions for the existence of an upper semicontinuous order-preserving function $u$ for a fuzzy binary relation $R$ on a crisp topological space $(X, \tau)$, which guarantees the existence of a maximal element for $R$ whenever $\tau$ is a compact topology.

The paper is structured as follows. Section 2 contains the notation and the preliminary results concerning crisp binary relations. Section 3 concerns the existence of an order-preserving function for fuzzy binary relations under decisiveness. In Section 4 we present a condition for the existence of an upper semicontinuous order-preserving function for a fuzzy binary relation on a crisp topological space. The conclusions are contained in Section 5.

\section{Notation and Preliminary Results on the Real Representation of Crisp Binary Relations}

We start with the presentation of the classical definitions and properties concerning the real representation of (crisp) binary relations, which are needed in this paper.

Definition 1. A binary relation $R$ on a nonempty crisp set $X$ is a (crisp) subset of the Cartesian product $X \times X$; i.e., a mapping $R: X \times X \mapsto\{0,1\}$. For any pair $(x, y) \in X \times X$, we write, for the sake of simplicity, $x R y$ instead of $(x, y) \in R . x R y$ has to be read as "the alternative $x \in X$ is at least as good as the alternative $y \in X$ ", or equivalently " $x \in X$ is weakly preferred to $y \in X$ ".

For the sake of convenience, and since our agreement on the interpretation of the binary relation $R$, from now on we shall denote by $\succsim$ a (crisp) binary relation on a set $X$. The following definitions are found, for example, in Herden and Levin [8].

Definition 2. The strict relation $\succ$, the indifference relation $\sim$, and the incomparability relation $\bowtie$ associated to a binary relation $\succsim$ on a set $X$ are defined as follows for all $x, y \in X$ :

(i) $x \succ y \Leftrightarrow(x \succsim y)$ and $\operatorname{not}(y \succsim x)$;

(ii) $x \sim y \Leftrightarrow(x \succsim y)$ and $(y \succsim x)$;

(iii) $\quad x \bowtie y \Leftrightarrow \operatorname{not}(x \succsim y)$ and $\operatorname{not}(y \succsim x)$.

The following definition of transitive closure is found, for example, in Suzumura [14]. 
Definition 3. The transitive closure $\succsim^{T}$ of a binary relation $\succsim$ on a set $X$ is defined as follows for every $x, y \in X:$

$$
x \succsim^{T} y \Leftrightarrow \exists n \in \mathbb{N}^{+}, \exists x_{0}, x_{1}, \ldots, x_{n} \in X \text { such that }\left[x=x_{0} \succsim x_{1} \succsim \ldots \succsim x_{n}=y\right]
$$

Definition 4. Consider a binary relation $\succsim \subset X \times X$. Then $\succsim$ is said to be:

(i) reflexive if $x \succsim x$ for all $x \in X$;

(ii) transitive if $(x \succsim y)$ and $(y \succsim z)$ imply $x \succsim z$ for all $x, y, z \in X$;

(iii) Suzumura consistent if for all $x, y \in X, x \succsim^{T} y$ implies not $(y \succ x)$;

(iv) a preorder if $\succsim$ is reflexive and transitive;

(v) a quasi-preorder if $\succsim$ is reflexive and Suzumura consistent;

(vi) $\quad$ total if $(x \succsim y)$ or $(y \succsim x)$ for all $x, y \in X$.

Please notice that all the above definitions are standard and very well-known in utility theory (see e.g., Herden [9]), despite for the concept of a quasi-preorder. Indeed, Suzumura consistency was introduced by Suzumura [14] in order to generalize Szpilrajn theorem (see Szpilrajn [15]) by introducing a condition which is more general than the classical transitivity assumption, but it does not appear specifically in connection with the real representation of binary relations (see Example 1 below).

Definition 5. Given a quasi-preorder $\succsim$ on a set $x$, let us define, for every point $x \in X$, the following subsets of $X$ :

$$
\begin{array}{ll}
l_{\succsim}(x)=\{y \in X \mid x \succ y\}, & r_{\succsim}(x)=\{y \in X \mid y \succ x\} \\
d_{\succsim}(x)=\{z \in X \mid x \succsim z\}, & i_{\succsim}(x)=\{z \in X \mid z \succsim x\} .
\end{array}
$$

We say that $l_{\succ}(x)\left(r_{\succ}(x)\right)$ is the strict lower section (respectively, strict upper section) associated to $x \in X$, while $d_{\succsim}(x)\left(i_{\succsim}(x)\right)$ is the weak lower section (respectively, weak upper section) associated to $x \in X$ (see e.g., Bridges and Mehta [7]).

Definition 6. A subset $D$ of a quasi-preordered set $(X, \succsim)$ is said to be $\succsim$-decreasing if $d \succsim(x) \subset D$ for all $x \in D$.

In the sequel, $\mathbb{R}$ stands for the real line, and $\geq$ is the natural (total) order on $\mathbb{R}$.

Definition 7. Consider a binary relation $\succsim$ on a set $X$, and a mapping $u:(X, \succsim) \mapsto(\mathbb{R}, \geq)$. Then $u$ is said to be:

(i) $\succsim$-increasing, if for all pairs $(x, y) \in X \times X,[x \succsim y \Rightarrow u(x) \geq u(y)]$;

(ii) a weak utility for $\succ$, if for all pairs $(x, y) \in X \times X,[x \succ y \Rightarrow u(x)>u(y)]$;

(iii) $\succsim$-order-preserving, if $u$ is both $\succsim$-increasing and a weak utility for $\succ$;

(iv) a utility for $\succsim$, if for all pairs $(x, y) \in X \times X,[x \succsim y \Leftrightarrow u(x) \geq u(y)]$.

Let us recall the concept of a maximal element for a (quasi-)preorder (see e.g., Bosi and Zuanon [20]).

Definition 8. Let $\succsim$ be a quasi-preorder on a set $X$. Then an element $x_{0} \in X$ is said to be a maximal element for $\succsim$ if for no $z \in X$ it happens that $z \succ x_{0}$.

It is immediate to check that a binary relation admitting a utility (representation) is a total preorder. The distinction between the concept of $\mathrm{a} \succsim$-order-preserving function and that of a weak utility for the strict part $\succ$ of $\succsim$ proved to be useful in connection with the existence of maximal elements of a preorder (see Bosi and Zuanon [20]).

It is very well known that the existence of an order-preserving function $u$ does not characterize a nontotal (quasi-)preorder $\succsim$, since whenever $u(x)>u(y)$ it may happen that either $(x \succ y)$ or 
$(x \bowtie y)$. Nevertheless, it is enough for optimization purposes; i.e., in order to guarantee the existence of maximal elements (see e.g., the introduction in Alcantud et al. [21]). More generally, it is clear that if $u$ is a weak utility for the strict part $\succ$ of a quasi-preorder $\succsim$ on a set $X$, and $u$ attains its maximum at a point $x_{0}$, then $x_{0}$ is a maximal element for $\succsim$.

Let us consider the following very simple proposition, which illustrates the importance of Suzumura consistency in connection with the existence of $\succsim$-order-preserving functions.

Proposition 1. If there exists a $\succsim$-order-preserving function $u:(X, \succsim) \mapsto(\mathbb{R}, \geq)$ for a (reflexive) binary relation $\succsim$ on a set $X$, then $\succsim$ is Suzumura consistent (a quasi-preorder).

Proof. Let $u:(X, \succsim) \mapsto(\mathbb{R}, \geq)$ be a $\succsim$-order-preserving function for a (reflexive) binary relation $\succsim$ on a set $X$. By contraposition, assume that $\succsim$ is not Suzumura consistent. Then there exist $n \in \mathbb{N}^{+}$, and elements $x_{0}, x_{1}, \ldots, x_{n} \in X$ such that $\left(x=x_{0} \succsim x_{1} \succsim \ldots \succsim x_{n}=y\right)$ and $(y \succ x)$, implying that $u(x)=u\left(x_{0}\right) \geq u\left(x_{1}\right) \geq \ldots \geq u\left(x_{n}\right)=u(y)>u(x)$, a contradiction.

The following example shows that the existence of a $\succsim$-order-preserving function $u:(X, \succsim) \mapsto(\mathbb{R}, \geq)$ does not imply that $\succsim$ is a preorder (in particular, does not imply that $\succsim$ is transitive) on $X$.

Example 1. Consider the binary relation $\succsim$ on the real interval $X=[0,1]$ defined as follows:

$$
x \succsim y \Leftrightarrow\left\{\begin{array}{l}
(x \geq y) \text { and }\left(x, y \in\left[0, \frac{1}{2}\right]\right) \\
\text { or } \\
(x \geq y) \text { and }\left(x, y \in\left[\frac{1}{2}, 1\right]\right) \\
\text { or } \\
\left(x \geq \frac{3}{4}\right) \text { and }\left(y \leq \frac{1}{4}\right)
\end{array}\right.
$$

It is clear that $\succsim$ is reflexive. Notice that $x \bowtie y$ for all $x, y \in[0,1]$ such that $\frac{3}{4}>x \geq \frac{1}{2}>y>\frac{1}{4}$. We have that $\succsim$ is not transitive since, for example, $\frac{3}{5} \succsim \frac{1}{2} \succsim \frac{1}{3}$ but $\frac{3}{5} \bowtie \frac{1}{3}$. On the other hand, it is easily seen that the identity function id $[0,1]$ on $[0,1]$ is a consistent (actually a quasi-preorder) on $[0,1]$ by Proposition 1.

The following concept of an extension (or refinement) of a binary relation by a total preorder is well known since the seminal work of Szpilrajn [15].

Definition 9. A total preorder $\succsim *$ is said to be an extension of a quasi-preorder $\succsim$ on a set $X$ if, for all $x, y \in X$, $\left[\left(x \succsim y \Rightarrow x \succsim^{*} y\right)\right.$ and $\left.\left(x \succ y \Rightarrow x \succ^{*} y\right)\right]$.

The following concept of separability is found, for example, in Herden and Levin [8].

Definition 10. A quasi-preorder $\succsim$ on a set $X$ is said to be separable if there exists a countable subset $D$ of $X$ such that, for all pairs $(x, y) \in X \times X$ such that $x \succ y$, there exists $d \in D$ such that $x \succsim d \succsim y$.

The following proposition provides an immediate characterization of the existence of $\mathrm{a} \succsim$-order-preserving function in terms of the existence of a representable (i.e., admitting a utility function $u:(X, \succsim) \mapsto(\mathbb{R}, \geq))$ total preorder ${ }^{*}$ providing an extension of $\succsim$. Please notice that in the sequel, the symbol $\succsim$ will stand for a quasi-preorder on a (nonempty) set $X$.

Proposition 2. The following conditions are equivalent on a quasi-preorder $\succsim$ on a set $X$ :

(i) There exists $a \succsim$-order-preserving function $u:(X, \succsim) \mapsto(\mathbb{R}, \geq)$;

(ii) There exists a representable total preorder ${ }^{*}$ which is an extension of $\succsim$;

(iii) There exists a total preorder $\succsim *$ which is a separable extension of $\succsim$. 
Proof. The equivalence of conditions (ii) and (iii) is well known (see e.g., Theorem 1.4.8 in Bridges and Mehta [7]). Therefore, we only have to show the equivalence of conditions (i) and (ii). In order to show that (i) implies (ii), just consider that if $u:(X, \succsim) \mapsto(\mathbb{R}, \geq)$ is a $\succsim$-order-preserving function, the representable total preorder $\succsim^{*}$ on $X$ defined as follows for all $x, y \in X:\left[x \succsim^{*} y \Leftrightarrow u(x) \geq u(y)\right]$ is an extension of $\succsim$. In order to show that (ii) implies (i), let $\succsim *$ be a representable extension of $\succsim$, and let $u$ be a utility function for $\succsim^{*}$. Then, it is immediate to check that $u$ is in particular a $\succsim$-order-preserving function. This consideration completes the proof.

Conditions for the existence of an upper semicontinuous and respectively continuous order-preserving function $u$ for a preorder $\succsim$ on a set $X$ endowed with a (crisp) topology $\tau$ are found, for example, in Bosi and Mehta [12].

Corollary 1. Let $\succsim$ be a reflexive binary relation on a countable set $X$. Then the following conditions are equivalent:

(i) There exists $a \succsim$-order-preserving function $u:(X, \succsim) \mapsto(\mathbb{R}, \geq)$;

(ii) $\succsim$ is a quasi-preorder.

Proof. The implication "(i) $\Rightarrow$ (ii)" is a consequence of Proposition 1. The implication "(ii) $\Rightarrow$ (i)" is a consequence of Proposition 2, implication "(iii) $\Rightarrow$ (i)", and of Suzumura's extension theorem [14] (see also Theorem 2 in Cato [17]), which states that a binary relation $\succsim$ is Suzumura consistent if and only if it admits an extension by means of a total preorder $\succsim^{*}$. On the other hand, such a total preorder $\succsim *$ is separable since $X$ is a countable set, and therefore it is representable by means of a utility function $u$, which is clearly a $\succsim$-order-preserving function.

\section{Order-Preserving Functions for Fuzzy Binary Relations under Decisiveness}

We first recall the classical concept of a fuzzy binary relation $R$, in order to then introduce the definition of its strict part under decisiveness $P_{R}$.

Definition 11. A fuzzy subset $h$ of a nonempty crisp set $X$ is a mapping $h: X \mapsto[0,1]$ from $X$ into the closed bounded real interval $[0,1]$. We denote by $F(X)$ the set of all the fuzzy subsets of $X$.

Definition 12. A fuzzy binary relation $R$ on a nonempty crisp set $X$ is a fuzzy subset of the Cartesian product $X \times X$; i.e. a mapping $X \times X \mapsto[0,1]$. For any pair $(x, y) \in X \times X, R(x, y)$ has to be thought of as "the degree to which the alternative $x \in X$ is at least as good as the alternative $y \in X^{\prime \prime}$. We write $R \in F(X, X)$.

Definition 13. Consider $R \in F(X, X)$. Then the strict part $P_{R}$ of $R\left(P_{R} \in F(X, X)\right)$ is defined to be, for all $x, y \in X$

$$
P_{R}(x, y):= \begin{cases}R(x, y) & \text { if }(R(x, y)>R(y, x)) \text { and }(R(x, y)+R(y, x) \geq 1) \\ 0 & \text { otherwise }\end{cases}
$$

Therefore, for any pair $(x, y) \in X \times X, P_{R}(x, y)$ has to be thought of as "the degree to which the alternative $x \in X$ is strictly preferred to the alternative $y \in X$ with decisiveness".

Remark 1. Our definition of the strict part $P_{R}$ of a fuzzy binary relation $R$ takes into account the possibility of dealing with nontotal fuzzy relations $R$ on $X$ (i.e., when there exist $x, y \in X$ such that $R(x, y)+R(y, x)<1$ ), in such a way that "decisiveness applies" (i.e., only pairs at which $R$ is decisive are considered as important). Clearly, when we consider total binary relations (preorders) (i.e. when $R(x, y)+R(y, x) \geq 1$ for all $x, y \in X$ ), the above definition coincides with the usual definition of the strict part of a binary relation, as found for example in Fono and Salles [3]. 
Definition 14. A fuzzy binary relation $R \in F(X, X)$ is said to be decisive at a pair $(x, y) \in X \times X$ if $R(x, y)+R(y, x) \geq 1$.

Let us now define the binary relation $\succsim_{R}$ on $X$ which is naturally associated to a fuzzy binary relation $R$ on $X$ in the case when "decisiveness" applies.

Definition 15. Let $R: X \times X \mapsto[0,1]$ be a fuzzy binary relation on a set $X$. Then the binary relation $\succsim_{R}$ on $X$ which is naturally associated to $R$ in the case of decisiveness is defined to be, for all $x, y \in X$,

$$
x \succsim_{R} y \Leftrightarrow(R(x, y) \geq R(y, x)) \text { and }(R \text { is decisive at }(x, y)) \text {. }
$$

Definition 16. Consider $R \in F(X, X)$. Then $R$ is said to be:

(i) reflexive if $R(x, x)=1$ for all $x \in X$;

(ii) min-transitive if $R(x, z) \geq \min \{R(x, y), R(y, z)\}$ for all $x, y, z \in X$;

(iii) bin-transitive if $(R(x, y) \geq R(y, x)$ and $(R(y, z) \geq R(z, y))$ imply $R(x, z) \geq R(z, x)$ for all $x, y, z \in X$;

(iv) fuzzy decisively Suzumura consistent if for every positive integer $n$, and for all elements $x_{0}, x_{1}, \ldots, x_{n} \in X$ such that $R$ is decisive at $\left(x_{h}, x_{h+1}\right)$ for all $h \in\{0, \ldots n-1\}$,

$$
\left[R\left(x_{h}, x_{h+1}\right) \geq R\left(x_{h+1}, x_{h}\right) \text { for all } h \in\{0, \ldots n-1\}\right] \Rightarrow P_{R}\left(x_{n}, x_{0}\right)=0
$$

(v) a fuzzy preorder if $R$ is reflexive and min-transitive;

(vi) a fuzzy decisive quasi-preorder if $R$ is reflexive and fuzzy decisively Suzumura consistent;

(vii) total if $R$ is decisive at every point $(x, y) \in X \times X$.

The reader can notice that all the previous definitions are classical and frequently found in the literature (perhaps with slightly different terminologies), except for the fuzzy decisive Suzumura consistency. In particular the term "total" is replaced by "connected" in Fono and Andjiga [2] and Fono and Salles [3].

Remark 2. In the particular case when $R: X \times X \mapsto\{0,1\}$ is a (crisp) binary relation on the set $X$ (i.e., $R \subset X \times X$ ), axioms (i), (ii) (equivalently (iii)) and (iv) in Definition 16 become axioms (i), (ii), and respectively (iii) in Definition 4.

Remark 3. It should be noted that $\succsim_{R}$ is not transitive in general, even if there exists $a \succsim_{R}$-order-preserving function $u$ on $X$. Further, $\succsim_{R}$ is reflexive if and only if the fuzzy binary relation $R \in F(X, X)$ is reflexive. On the other hand, it is clear that if $R \in F(X, X)$ is total, then the associated binary relation $\succsim_{R}$ in Definition 15 is the total preorder $\succsim_{R}$ on $X$ defined as follows for all $x, y \in X$ :

$$
x \succsim_{R} y \Leftrightarrow R(x, y) \geq R(y, x)
$$

This is precisely the case of the utility representation of a fuzzy total preorder considered in Billot [1], Fono and Andjiga [2], and Fono and Salles [3].

Remark 4. It is immediate to observe that a reflexive binary relation $R \in F(X, X)$ is a fuzzy quasi-preorder if and only if the associated binary relation $\succsim_{R}$ in Definition 15 is a quasi-preorder according to Definition 4, (v).

Definition 17. Consider a fuzzy binary relation $R \in F(X, X)$, and mapping $u:(X, R) \mapsto(\mathbb{R}, \geq)$. Then $u$ is said to be:

(i) $R$-increasing, if, for all pairs $(x, y) \in X \times X$ at which $R$ is decisive, $[R(x, y) \geq R(y, x) \Rightarrow u(x) \geq u(y)]$;

(ii) a weak utility for $P_{R}$, if for all pairs $(x, y) \in X \times X,\left[P_{R}(x, y)>0 \Rightarrow u(x)>u(y)\right]$;

(iii) $R$-order-preserving, if $u$ is both $R$-increasing and a weak utility for $P_{R}$;

(iv) a utility for $R$ if $R$ is total and, for all pairs $(x, y) \in X \times X,[R(x, y) \geq R(y, x) \Leftrightarrow u(x) \geq u(y)]$. 
It should be noted that the terminology above is perfectly coherent with the usual one recalled in Definition 7 concerning crisp relations (preorders), since in this case $R(x, y)+R(y, x) \geq 1$ is equivalent to $\max (R(x, y), R(y, x))=1$.

The following definition of separability of a fuzzy binary relation is found in Fono and Salles [3].

Definition 18. A fuzzy binary relation $R \in F(X, X)$ is said to be separable if there exists a countable subset $D$ of $X$ such that, for all pairs $(x, y) \in X \times X$ at which $R$ is decisive and $R(x, y)>R(y, x)$ there exists $d \in D$ such that $R$ is decisive both at $(x, d)$ and $(d, y)$ and $R(x, d) \geq R(d, x), R(d, y) \geq R(y, d)$.

As an immediate consequence of the above definitions and Proposition 2, we get the following proposition, whose immediate proof is left to the reader.

Proposition 3. The following conditions are equivalent on fuzzy binary relation $R \in F(X, X)$ and a real-valued function $u$ on $X$ :

(i) $\quad u:(X, R) \mapsto(\mathbb{R}, \geq))$ is an R-order-preserving function;

(ii) $\left.\quad u:\left(X, \succsim_{R}\right) \mapsto(\mathbb{R}, \geq)\right)$ is a $\succsim_{R}$-order-preserving function, where $\succsim_{R}$ is the binary relation naturally associated to $R$ according to Definition 15;

(iii) $\quad u$ is the utility function for some total preorder $\succsim^{*}$ which is an extension of $\succsim_{R}$.

Proposition 4. Consider a fuzzy binary relation $R \in F(X, X)$. Then there exists an $R$-order-preserving function $u:(X, R) \mapsto(\mathbb{R}, \geq)$ provided that there exists a fuzzy total preorder $R^{*}: X \times X \mapsto[0,1]$ satisfying the following conditions:

(i) $R^{*}$ is separable;

(ii) The following conditions are verified for all $(x, y) \in X \times X$ at which $R$ is decisive,

(a) $\quad R(x, y) \geq R(y, x)$ implies $R^{*}(x, y) \geq R^{*}(y, x)$;

(b) $\quad P_{R}(x, y)>0$ implies $P_{R^{*}}(x, y)>0$;

(iii) For every $x, y, z \in X, R^{*}(x, y)=R^{*}(y, x)=R^{*}(y, z)=R^{*}(z, y) \Rightarrow R^{*}(x, z)=R^{*}(z, x)$.

Proof. Assume that there exists a fuzzy total preorder $R^{*}: X \times X \mapsto[0,1]$ satisfying the above conditions (i), (ii), and (iii). Then, from Proposition 2 in Fono and Salles [3], since conditions (i) and (iii) hold true, there exists a utility function $u:\left(X, R^{*}\right) \mapsto(\mathbb{R}, \geq)$. Such utility function $u$ is $R$-order-preserving by condition (ii). This consideration completes the proof.

The following theorem provides a characterization of the existence of an $R$-order-preserving function for a fuzzy binary relation $R \in F(X, X)$ in case that the set $X$ is countable.

Theorem 1. Consider a fuzzy binary relation $R \in F(X, X)$. If $R$ is reflexive and $X$ is countable, then the following conditions are equivalent:

(i) There exists an $R$-order-preserving function $u:(X, R) \mapsto(\mathbb{R}, \geq)$;

(ii) $R$ is a fuzzy decisive quasi-preorder.

Proof. (i) $\Rightarrow$ (ii). If there exists an order-preserving function $u:(X, R) \mapsto(\mathbb{R}, \geq)$, then, by Proposition $\left.3, u:\left(X, \succsim_{R}\right) \mapsto(\mathbb{R}, \geq)\right)$ is an $\succsim_{R}$-order-preserving function, where $\succsim_{R}$ is the binary relation naturally associated to $R$ according to Definition 15 . Hence, we have that $\succsim_{R}$ is a quasi-preorder by Proposition 2, and therefore $R$ is a fuzzy decisive quasi-preorder from the definition of $\succsim_{R}$.

(ii) $\Rightarrow$ (i). If $R \in F(X, X)$ is a fuzzy decisive quasi-preorder, then $\succsim_{R}$ is a quasi-preorder, and therefore there exists a $\succsim_{R}$-order-preserving function by Corollary 1 . Clearly, $u$ is also $R$-order-preserving. This consideration completes the proof. 
Since for a fuzzy total binary relation $R \in F(X, X)$ a function $u:(X, R) \mapsto(\mathbb{R}, \geq)$ is $R$-order-preserving if and only if it is a utility function for $R$, we immediately get as a corollary the following result proved by Billot [1] (see also Proposition 2 in Fono and Andjiga [2]).

Corollary 2. Consider a fuzzy total binary $R \in F(X, X)$. If $X$ is countable, then the following conditions are equivalent:

(i) There exists a utility function $u:(X, R) \mapsto(\mathbb{R}, \geq)$;

(ii) $R$ is bin-transitive.

\section{Upper Semicontinuous Order-Preserving Functions for Fuzzy Binary Relation on A Topological Space}

In order to motivate the contents of this section, let us first introduce the concept of a maximal element for a fuzzy binary relation under decisiveness.

Definition 19. A point $x_{0} \in X$ is said to be maximal with respect to fuzzy binary relation $R \in F(X, X)$ if for no $z \in X$ it occurs that $P_{R}\left(z, x_{0}\right)>0$ (i.e., $r_{\succsim_{R}}\left(x_{0}\right)=\varnothing$ ).

It is clear that if a binary relation $R \in F(X, X)$ admits a representation by means of an $R$-order-preserving $u$, and $u$ attains its maximum at a point $x_{0} \in X$, then $x_{0}$ is a maximal element for $R$. In the sequel, the symbol $\succsim_{R}^{T}$ stands for the transitive closure of the binary relation $\succsim_{R}$ associated to some $R \in F(X, X)$.

Definition 20. Let $R \in F(X, X)$ be a fuzzy binary relation on a set $X$, endowed with a topology $\tau$. Then $R$ is said to be:

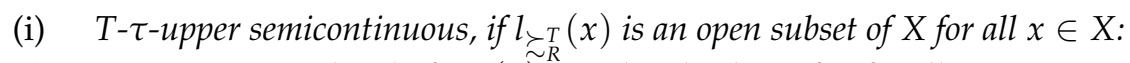

(ii) T- $\tau$-upper semiclosed, if $i_{\downarrow_{R}}(x)$ is a closed subset of $X$ for all $x \in X$.

It should be noted that if $R$ is a crisp binary relation, then the above definitions of $T$ - $\tau$-upper semicontinuity and $T$ - $\tau$-upper semiclosedness slightly generalize the concepts of upper semicontinuity of type 1 and respectively upper semicontinuity of type 2 as defined in Herden and Levin [8] in the case of a preorder $\succsim$ on a topological space $(X, \tau)$.

We recall that a (crisp) topology $\tau$ on a set $X$ is a family of subsets of $X$ which contains $X$ and the empty set $\varnothing$ and is closed under arbitrary unions and finite intersections. A set $O \in \tau$ is said to be open. We denote by $\tau_{\text {nat }}$ the natural (interval) topology on $\mathbb{R}$. Further, a real-valued function $u$ on a topological space $(X, \tau)$ is said to be $\tau$-upper semicontinuous if the set $u^{-1}(]-\infty, \alpha[)=\{z \in X: u(z)<\alpha\}$ is open for every $\alpha \in \mathbb{R}$.

Proposition 5. Let $R$ be a fuzzy decisive quasi-preorder on a set $X$, endowed with a topology $\tau$. Then there exists a $\tau$-upper semicontinuous $R$-order-preserving function $u:(X, R, \tau) \mapsto\left(\mathbb{R}, \geq, \tau_{\text {nat }}\right)$ provided that the following conditions are verified:

(i) There exists a countable subset $D$ of $X$ such that for all pairs $(x, y) \in X \times X$ such that $x \succ_{R} y$, there exists $d \in D$ such that $x \succsim_{R} d \succ_{R}^{T} y$;

(ii) $\quad R$ is either $T$ - $\tau$-upper semicontinuous or $T$ - $\tau$-upper semiclosed.

Proof. Let $R$ be a fuzzy decisive quasi-preorder on a set $X$, endowed with a topology $\tau$, and assume that $R$ satisfies the above conditions (i) and (ii). Let $D=\left\{d_{n}: n \in \mathbb{N}^{+}\right\}$be a countable subset of $X$ verifying the above condition (i), and define the function $u_{n}: X \mapsto[0,1]_{\mathbb{R}}$ as follows for every $n \in \mathbb{N}^{+}$:

$$
u_{n}(x)=\left\{\begin{array}{ll}
0 & \text { if } x \in l_{\substack{\downarrow_{R} \\
(}}\left(d_{n}\right)\left(x \in X \backslash i_{\succsim_{R}}\left(d_{n}\right)\right) . \\
1 & \text { otherwise }
\end{array} .\right.
$$


Then define $u:=\sum_{n=1}^{\infty} 2^{-n} u_{n}$. Observe that $u$ is $\succsim_{R}$-increasing since the fact that $l_{\succsim_{R}^{T}}(z)\left(X \backslash i_{\succsim_{R}^{T}}(z)\right)$ is $\succsim_{R}^{T}$-decreasing for all $z \in X$ implies that $u_{n}$ is $\succsim_{R}^{T}$-increasing for all $n \in \mathbb{N}^{+}$, and therefore that $u$ is $\succsim_{R}$-increasing. Further, it is easily seen that the above condition (i) implies that $u$ is a weak utility for $\succ_{R}$. Indeed, if $x \succ_{R} y$, then there exists $n \in \mathbb{N}^{+}$such that $x \succsim_{R} d_{n} \succ_{R}^{T} y$, implying that $u_{n}(y)=0$ and $u_{n}(x)=1$. Since $R$ is $T$ - $\tau$-upper semicontinuous (T- $\tau$-upper semiclosed), it is easy to be seen that $u_{n}$ is $\tau$-upper semicontinuous for all $n \in \mathbb{N}^{+}$, implying that $u$ is also $\tau$-upper semicontinuous. This consideration completes the proof.

Since every upper semicontinuous function $u$ attains its maximum on a compact topological space $(X, \tau)$ (i.e., when for every family $\mathcal{O}=\left\{O_{\alpha}\right\}_{\alpha \in I}$ of open subsets of $X$ such that $\bigcup_{\alpha \in I} O_{\alpha}=X$ there exists a finite subfamily $\mathcal{O}^{\prime}=\left\{O_{1}, \ldots, O_{n}\right\}$ of $\mathcal{O}$ such that $\bigcup_{n=1}^{n} O_{n}=X$ ), we immediately get the following corollary.

Corollary 3. Let $R$ be a fuzzy decisive quasi-preorder on a set $X$, endowed with a topology $\tau$. If $R$ satisfies the assumptions of Proposition 5, and the topology $\tau$ is compact, then there exists a maximal element $x_{0}$ for $R$.

\section{Conclusions}

In this paper we have presented conditions for the existence of an order-preserving function $u$ for a fuzzy not necessarily total binary relation $R$ on a set $X$ when decisiveness applies, in the sense that $u$ is required to "preserve" and "to strictly preserve" the inequalities $R(x, y) \geq R(y, x)$ and respectively $R(x, y)>R(y, x)$ only in the case of a pair $(x, y) \in X \times X$ such that $R(x, y)+R(y, x) \geq 1$ (i.e., when $R$ is decisive at $(x, y)$ according to the terminology adopted here). By using this approach, it is possible to generalize the corresponding results concerning the existence of a utility function $u$ for a fuzzy total preorder $R$ on $X$; i.e., when $R(x, y)+R(y, x) \geq 1$ for all pairs $(x, y) \in X \times X$.

We have also presented conditions implying the existence of an upper semicontinuous order-preserving function in case that the set $X$ is endowed with a crisp topology.

The possibility of characterizing the existence of an (upper semicontinuous or continuous) order-preserving function for a not necessarily total fuzzy preference relation under decisiveness will hopefully be examined in a future paper.

Author Contributions: The authors contributed equally to this work.

Conflicts of Interest: The authors declare no conflict of interest.

\section{References}

1. Billot, A. An existence theorem or fuzzy utility functions: A new elementary proof. Fuzzy Sets Syst. 1995, 74, 271-276.

2. Fono, L.A.; Andjiga, N.G. Utility function of fuzzy preferences on a countable set under max-*-transitivity. Soc. Choice Welf. 2007, 28, 667-683.

3. Fono, L.A.; Salles, M. Continuity of utility functions representing fuzzy preferences. Soc. Choice Welf. 2011, 37, 669-682.

4. Agud, L.; Catalan, R.G.; Diaz, S.; Indurain, E.; Montes, S. Numerical representability of fuzzy total preorders. Int. J. Comput. Intell. Syst. 2012, 5, 996-1009

5. Mishra, S.; Srivastava, R. Representability of fuzzy biorders and fuzzy weak orders. Int. J. Uncertain. Fuzziness Knowl.-Based Syst. 2016, 24, 9117-9935.

6. Beg, I.; Ashraf, F. Numerical representation of product transitive complete fuzzy orderings. Math. Comput. Model. 2011, 53, 9617-9623.

7. Bridges, D.S.; Mehta, G.B. Representations of Preference Orderings; Springer: Berlin, Germany, 1995. 
8. Herden, G., Levin, V.L. Utility representation theorems for Debreu separable preorders. J. Math. Econ. 2012, $48,148-154$.

9. Herden, G. On the existence of utility functions. Math. Soc. Sci. 1989, 17, 297-313.

10. Mehta, G.B. Some general theorems on the existence of order-preserving functions. Math. Soc. Sci. 1988, 15, 135-146.

11. Bosi, G.; Campíon, M.J.; Candeal, J.C.; Induráin, E.; Zuanon, M. Numerical isotonies of preordered semigroups through the concept of a scale. Math. Pannonica 2005, 16, 65-77.

12. Bosi, G.; Mehta, G.B. Existence of a semicontinuous or continuous utility function: A unified approach and an elementary proof. J. Math. Econ. 2002, 38, 311-328.

13. Bosi, G.; Zuanon, M. Conditions for the Upper Semicontinuous Representability of Preferences with Nontransitive Indifference. Theor. Econ. Lett. 2014, 4, 371-377.

14. Suzumura, K. Remarks on the theory of collective choice. Economica 1976, 43, 381-390.

15. Szpilrajn, E. Sur l'extension de l'ordre partiel. Fundam. Math. 1930, 16, 386-389.

16. Bosi, G.; Herden, G. On a Strong Continuous Analogue of the Szpilrajn Theorem and its Strengthening by Dushnik and Miller. Order 2005, 22, 329-342.

17. Cato, S. Szpilrajn, Arrow and Suzumura: Concise proofs of extensions theorems and an extension. Metroeconomica 2012, 63, 235-249.

18. Bosi, G.; Estevan, A.; Zuanon, M. Partial representations of orderings. Int. J. Uncertain. Fuzziness Knowl.-Based Syst. 2017, in press.

19. Khalid, A.; Beg, I. Incomplete Hesitant Fuzzy Preference Relations in Group Decision Making. Int. J. Fuzzy Syst. 2017, 19, 637-645.

20. Bosi, G.; Zuanon, M. Maximal elements of quasi upper semicontinuous preorders on compact spaces. Econ. Theory Bull. 2017, 5, 109-117.

21. Alcantud, J.C.R.; Bosi, G.; Zuanon, M. Richter-Peleg multi-utility representations of preorders. Theory Decis. 2016, 80, 443-450.

(C) 2017 by the authors. Licensee MDPI, Basel, Switzerland. This article is an open access article distributed under the terms and conditions of the Creative Commons Attribution (CC BY) license (http:/ / creativecommons.org/licenses/by/4.0/). 